\title{
APPLICATION DE LA SPECTROSCOPIE DE PERTES D'ÉNERGIE D'ÉLECTRONS A HAUTE TENSION A L'ANALYSE DE MICROPARTICULES DANS DES COUPES DE TISSUS BIOLOGIQUES
}

\author{
J.P. Berry, P. Galle, Y. Kihn*, G. Zanchi*, J. Sevely* et B. Jouffrey* \\ Laboratoire de Biophysique, Université du val de Marne, 6 me du Général \\ Sarrail, 94010 Créteil, France \\ *Laboratoire d'Optique Electronique du CNRS, 29 me Jeanne Marvig, B.P. 4347, \\ 31055 Toulouse Cedex, France
}

\begin{abstract}
Résumé : La spectroscopie de pertes d'énergie d'électrons à haute tension est appliquée pour la caractérisation de microparticules intracellulaires observées dans les coupes de tissus biologiques. Elle permet de montrer que ces microparticules correspondent à des dépôts contenant divers éléments : ici de l'aluminium, du gallium, du nickel et de l'uranium.
\end{abstract}

Abstract : High voltage ejectron energy loss spectroscopy has been used for the chemical anaTys is of smal1 intracellular particles observed in thin biological tissue sections. The technique has allowed the characterization of aluminium, gallium, nickel and uranium.

Associēe à l'observation des micrographies électroniques, la technique de spectroscopie de pertes d'énergie d'électrons est maintenant largement utîlisée comme méthode d'analyse chimique à 1 'échelle submicrométrique. L'expérience montre que l'utilisation de hautes tensions accélératrices $(>300 \mathrm{kV})$ des électrons primaires permet 1 'analyse d'êchantillons plus épais que ceux qui peuvent être étudiês aux tensions plus classiques de $100 \mathrm{kV}$. L'étude de la visibilité des signaux caractéristiques d'excitation de niveaux atomiques profonds exploités pour l'analyse met bien en évidence cet avantage (1). Elle montre, en particulier, que le contraste de ce signal par rapport au fond continu auquel il se superpose, varie en raison inverse du paramètre $t / \lambda$ épaisseur $t$ de l'échantilion mesurée en unité de libre parcours moyen $\lambda$ du processus de diffusion inélastique principal dans T'échantilion. $\lambda$ correspond en général au processus de diffusion par plasmons. Il est multiplié par un facteur de $1^{\prime}$ ordre de 3 entre 100 et $1000 \mathrm{kV}(2,3)$. I I faut s'attendre à un rapport du même ordre entre les épaisseurs des coupes qui peuvent être exploitēes à 1000 et $100 \mathrm{kV}$. L'épaisseur des coupes de préparations biologiques qui peuvent être analysées à 1000 kV par cette méthode, est de 1 'ordre de $250 \mathrm{~nm}$. Les effets de diffusions multiples dans l'échantillon font qu'au delà de $500 \mathrm{~nm}$, les spectres ne sont plus utilisables pour l'analyse.

Le mode de variation du contraste des distributions caractéristiques en fonction de $t / \lambda$ permet aussi de conclure à une amélioration de la visibilité des rajes pour un même échantillon, obśervé dans des conditions comparables lorsque la tension accēlératrice des électrons primaires augmente. Ainsi la valeur limite des pertes d'énergie utilisables devient plus grande. A $1000 \mathrm{kV}$, elle atteint $3000 \mathrm{eV}$.

La technique a été utilisēe pour la détection de divers types d'éléments, de numéros atomiques aussi différents que l'aluminium $(Z=13)$ et l'uranium $(Z=92)$, dans des coupes de différents tissus. Elle a étë appliquée à la caractérisation de microparticules intracellulaires en général constituées d'amas de fins granules dont la taille est de l'ordre de $10 \mathrm{~nm}$. Les coupes étudiées étaient d'une épaisseur de l'ordre de $200 \mathrm{~nm}$. La partie de 1'échantillon sélectionnée pour l'analyse correspondait à une aire de 1 'objet de 100 à $200 \mathrm{~nm}$ de diamètre. 
I - DETECTION DE L'ALUMINIUM DANS DES COUPES DE MACROPHAGES ALVEOLAIRES

L'observation en microscopie électronique classique de coupes de macrophages alvẽolaires fait apparaftre des inclusions intracellulaires de nature lysosomiale de quelques $\mathrm{nm}$ de diamètre (Fig. $1 \mathrm{~A}$ ). Le spectre de pertes d'énergie associé à cette partie de l'image permet de mettre en évidence la prêsence d'aluminium par la distribution caractéristique d'excitation du niveau $K$ dont le seuil apparait dans le spectre à $1540 \mathrm{eV}$ (Fig l B). Le spectre montre également que, dans les inclusions, l'aluminium est associé à du phosphore (seuil $\mathrm{K}$ à $2150 \mathrm{eV}$ ) et à̀ de 1 'oxygène (seuil $\mathrm{K}$ à $530 \mathrm{eV}$ ).

\section{II - MISE EN EVIDENCE DU GALLIUM (5)}

La répartition du gallium dans les cellules rénales a été recherchéedans les lysosomes de cellules tubulaires. Cet élément apparaît également sous forme d'amas de fins granules (Fig. 2A) et se caractérise dans le spectre par la distribution L23 dont le

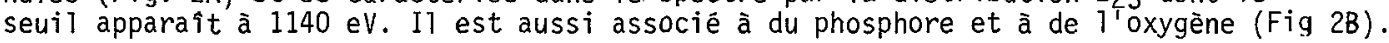

\section{III - CARACTERISATION DU NICKEL (6)}

La membrane de cellules tumorales en culture est soulignée par une précipitation dense (Fig. $3 \mathrm{~A}$ ). L'analyse par pertes d'énergie d'électrons a permis de montrer que cette structure correspond à une forte concentration en nickel -mis en évidence par son seuii $L_{23}$ à $810 \mathrm{eV}$ - associè à de l'oxygène, (Fig. $3 \mathrm{~B}$ ).

\section{IV - CARACTERISATION DE L'URANIUM (7)}

Les images de 7ysosomes de cellules tubulaires rénales font apparaitre des amas de fines aiguilles (Fig. 4 A). La nature chimique de ces amas, essentiellement constitués d'uranium, a pu être confirmēe par le spectre des pertes d'énergie. L'uranium est caractērisê par deux distributions - la distribution $\mathrm{N}_{45}$ à 730 ev et la distribution $\mathrm{N}_{23}$ à $1045 \mathrm{eV}$, (Fig. $4 \mathrm{~B}$ ).

\section{$V$ - CONCLUSION}

Nous avons donc tenté de préciser grâce à la spectrométrie des pertes d'énergie d'électrons utilisée a haute tension, la nature des inclusions submicroscopiques intracellulaires contenant des éléments minéraux très divers ( $A 1, N i, G a, U$ ) dans les différents tissus de mammifères' (rein, poumon, cellules tumoràles) ayant donné lieu à des protocoles expérimentaux très différents. Dans tous les cas étudiés, les élëments minéraux ont ètē mis en êvidence, parfois associēs à d'autres éléments confirmant un mécanisme de concentration et de précipitation dans les inclusions intracellulaires. La mise en évidence de très nombreux éTéments de la classification périodique offre donc de nouvelles perspectives dans l'étude des tissus biologiques. Dans le cas de cette étude, les donnêes fournies par cette méthode de microanalyse apportent de nouvelles connaissances dans le domaine de la pathologie cellulaire.

\section{REFERENCES}

(1) ZANCHI G., KIHN Y., SEVELY J., JOUFFREY B., Proc. 7th Inter. Conference on High Voltage Electron Microscopy, Berkeley (1983) 85.

- SEVELY J., KIHN Y., ZANCHI G., JOUfFREY B., these Proceedings.

(2) MISELL D.L., CRICK R.A., J. PhyS, C 2 (1969) 2290.

(3) SEVELY J., PEREZ J.Ph., JOUFFREY B., Proc 3rd Intern. Conference on High Voltage Electron Microscopy, Oxford(1974) 32.

(4) BERRY J.P., HENOC P., GALLE P., PARIENTE R., J. Microsc. 17 (1973) 11.

(5) BERRY J.P., GALLE S., ESCAIG F., these Proceedings.

(6) BERRY J.P., POUPON M.F., POT-DEPRUN J., Le Nickel dans 1 'environnement humain, Lyon, Mars 1983.

(7) GALLE P., J; Microsc. 19 (1974) 87. 


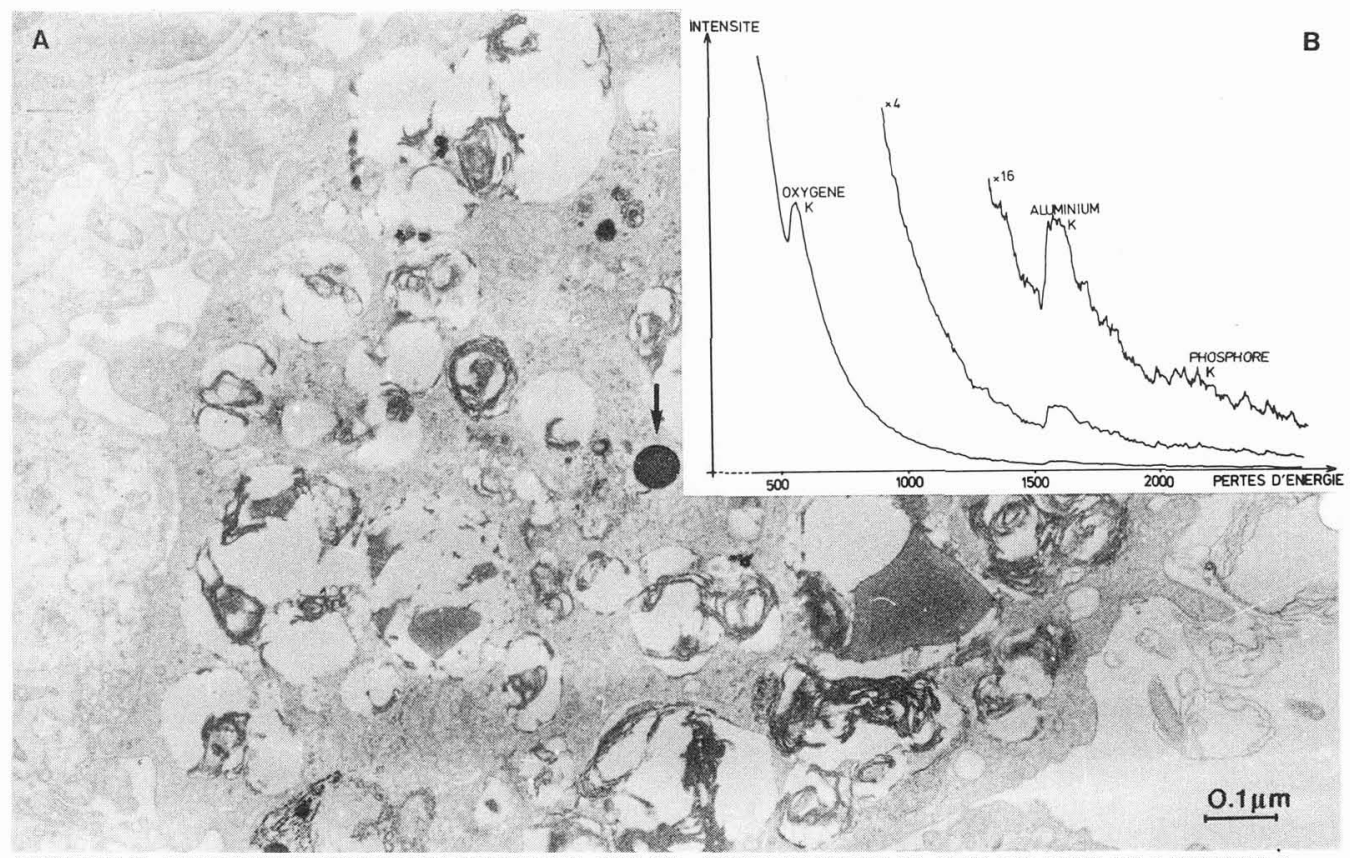

Fíg. 1 : A - Image en champ clair à $100 \mathrm{kV}$ de coupe de macrophages alvéolaires présentant des inclusions intracellulaires.

B - Spectre des pertes d'énergie associé à une de ces inclusions dans la zone des pertes $400-2500 \mathrm{eV}$ - mise en évidence des seuils de l'oxvaène, de l'aluminium et du phosshore.

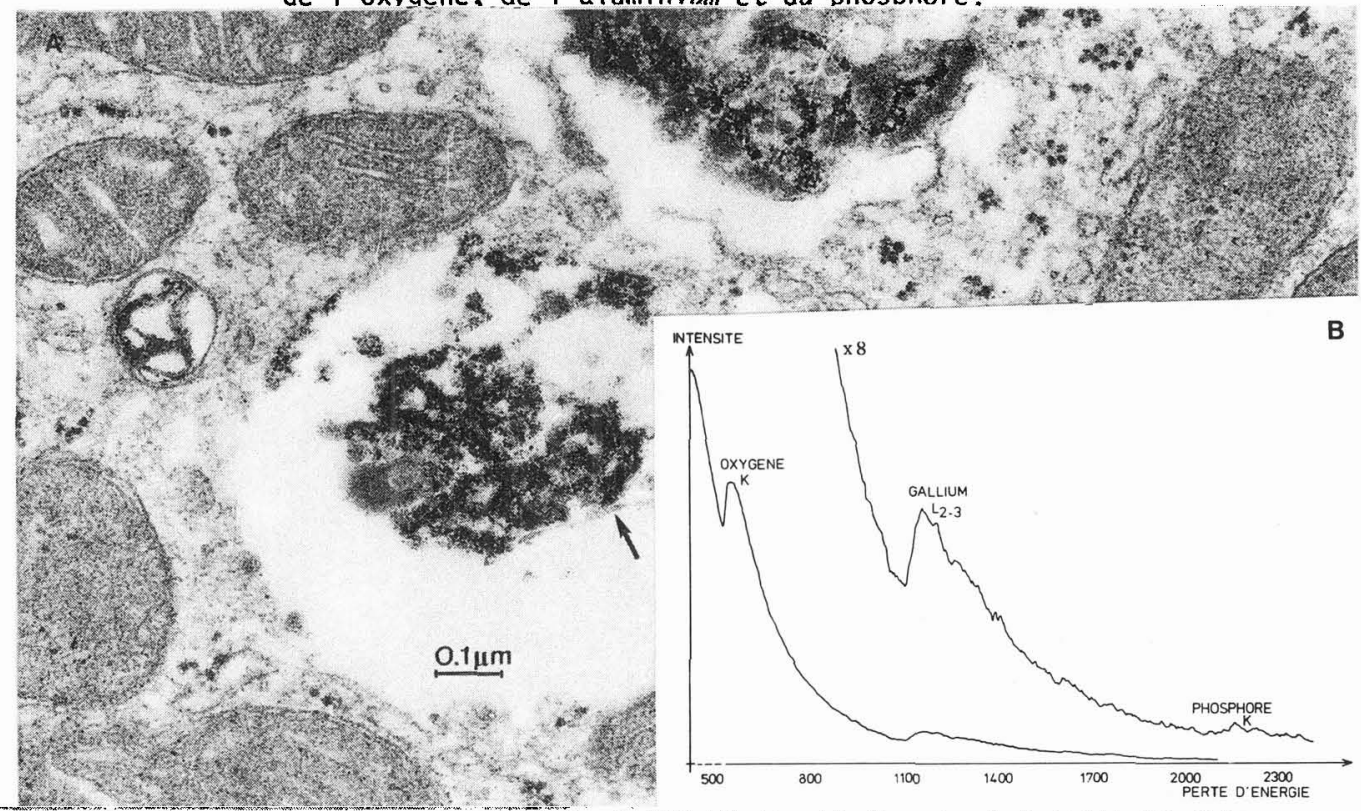

Fig. 2 : A - Image en champ Clair à $100 \mathrm{kV}$ d'amas de granules observés dans le lysosome de cellules tubulaires rénales.

B - Spectre des pertes d'énergie associé à cette zone - mise en évidence de 1'oxygène, du gallium et du phosphore. 


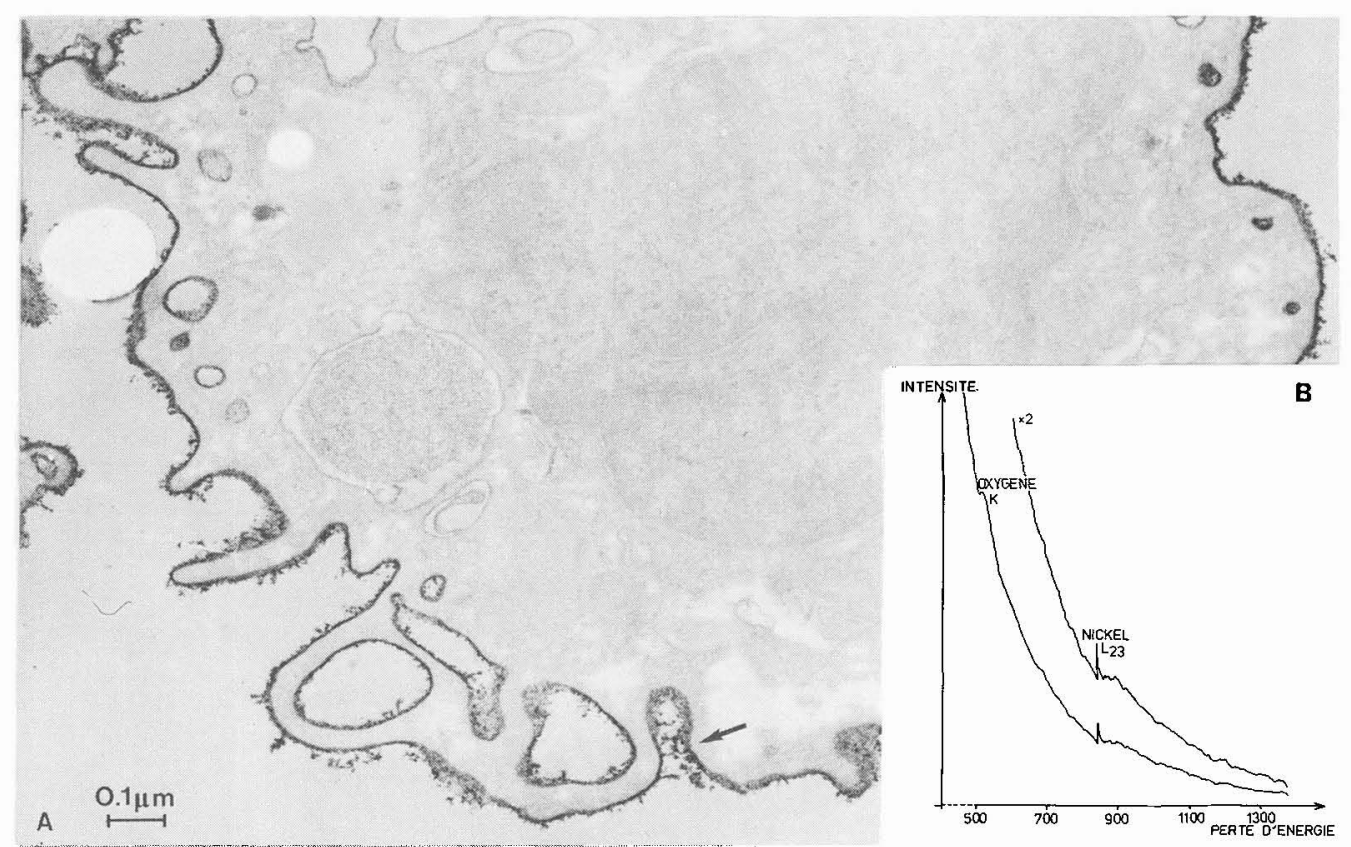

Fig. 3 : A - Image en champ clair à $100 \mathrm{kV}$ d'une portion de cellule tumorale. La membrane de la cellule apparaitt en noir.

B - Spectre de pertes d'énergie a 1 MV sur une portion de I'image contenant la membrane dans la bande de pertes d'énergie 400 - $1400 \mathrm{eV}$ mise en évidence de T'oxygène et du nickel.

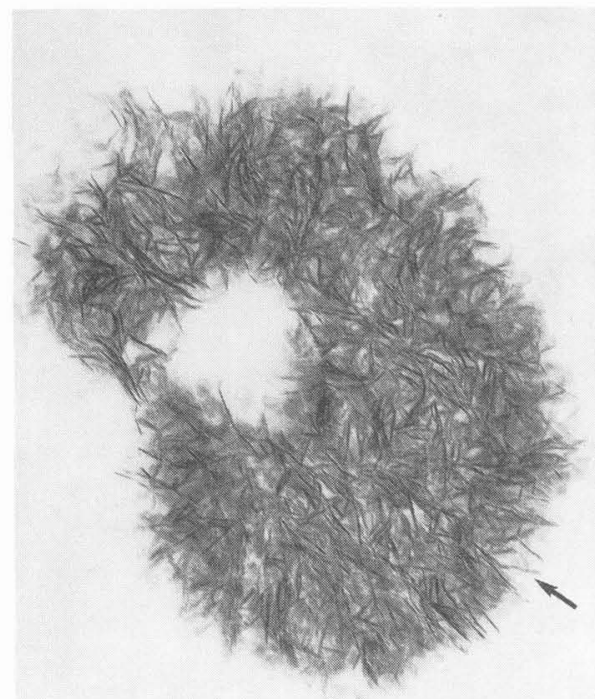

A $\stackrel{0.1 \mu \mathrm{m}}{\longmapsto}$

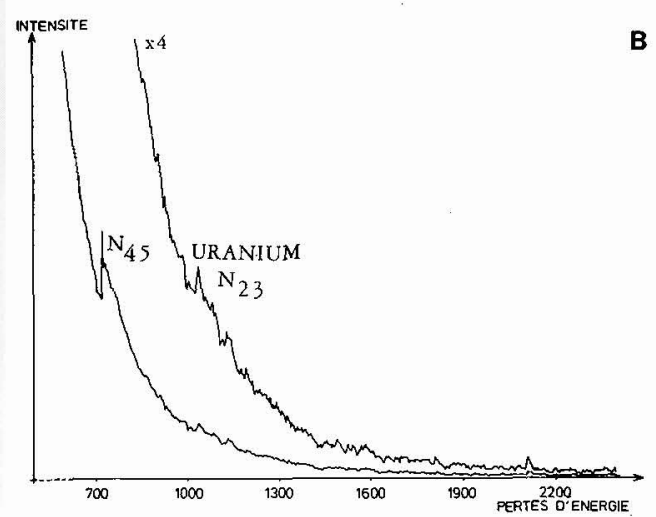

Fig. 4 : A - Image en champ clair à $100 \mathrm{kV}$ d'amas d'aiguilles à analyser dans des coupes de Tysosomes de cellules tubulaires.

$B$ - Spectre de pertes d'énergie obtenu à $1 \mathrm{MV}$ sur une portion de 1'image dans la fenêtre des pertes $600-2400 \mathrm{eV}$ - mise en évidence des seuils $\mathrm{N}_{23}$ et $\mathrm{N}_{45}$ de 1 'uranium. 